\title{
The success of various management techniques used in South African children with type 1 diabetes mellitus
}

\author{
K L Kalweit, ${ }^{1,2}$ BSc (Medical Sciences), BSc Hons (Bioinformatics); N Briers, ${ }^{3}$ BSc, BSc Hons (Physiology), PhD (Anatomy); \\ S A S Olorunju, ${ }^{4}$ BSc (Statistics), MSc (Mathematical Statistics), PhD (Applied Quantitative Genetics) \\ ${ }^{1}$ School of Health Systems and Public Health, Faculty of Health Sciences, University of Pretoria, South Africa \\ ${ }^{2}$ Youth With Diabetes, Johannesburg, South Africa \\ ${ }^{3}$ Department of Anatomy, Faculty of Health Sciences, University of Pretoria, South Africa \\ ${ }^{4}$ Biostatistics Unit, South African Medical Research Council, Pretoria, South Africa
}

Corresponding author: K Kalweit (k.kalweit@live.com)

Background. Despite the availability of international guidelines for the treatment of type 1 diabetes mellitus (T1DM) in children, important aspects of treatment are not accessible to all young patients in South Africa (SA).

Objective. To investigate factors in diabetes management strategies that are associated with poor glycaemic control and decreased quality of life (QoL) in SA children with T1DM.

Methods. Eighty children (mean (standard deviation) age 12.9 (2.7) years) with T1DM were asked to answer standardised questionnaires on demographics, management techniques used and perceptions of diabetes. The height and weight of each child was recorded and glycosylated haemoglobin (HbAlc) measured. Informed consent and assent for each participant was obtained before enrolment.

Results. A total of $51.4 \%$ of the participants had poor metabolic control, with an HbAlc level $>10.0 \%(86 \mathrm{mmol} / \mathrm{mol})$. Factors in clinical practice found to have a significant association with decreased HbA1c and/or QoL were healthcare system $(p<0.001)$, insulin administration $(p=0.001)$, correction dose $(p=0.002)$, carbohydrate counting $(p<0.001)$ and number of severe hyperglycaemic events $(p=0.048)$. Regular exercise did not show any association with HbA1c classification or QoL. Children from single-parent households were prone to unsuccessful diabetes management regardless of treatment techniques used $(p=0.002)$.

Conclusions. The use of premixed insulin without access to rapid-acting insulin, absence of correction doses for hyperglycaemia and lack of carbohydrate counting showed significant association with poor diabetes management. Some recommendations regarding the adoption of more effective diabetes management strategies in the public healthcare system are suggested.

S Afr Med J 2015;105(5):400-404. DOI:10.7196/SAMJ.9334

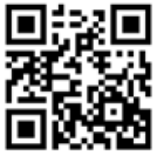

The current prevalence of diabetes in South Africa (SA) is $8.27 \%$, with an incidence rate of $0.8 / 100000$ for children aged $0-14$ years. ${ }^{[1]}$ The choice of treatment regimen for children living with type 1 diabetes mellitus (T1DM) should accommodate the child's age, daily routines, targets of metabolic control, and individual and family preferences. However, different strategies for T1DM management that may assist with these specific needs are not equally accessible in SA.

SA has a private and a public (funded) healthcare sector. Children cared for in the private setting have access to a wider range of insulin brands and more testing strips per month than their counterparts in the public system. Private patients also see diabetes educators and dieticians, although the frequency of such visits depends on their medical insurance scheme. Continuous subcutaneous insulin infusion (CSII) pumps are available in SA, but are available only to children on upper-tier insurance schemes owing to the high cost of the machine and its consumables.

Insulin is issued by the state to those not covered by medical insurance. ${ }^{[2]}$ However, because test strips for glucometers are not on national tender, availability depends on the individual budget of each public healthcare facility. Diabetes educators are not routinely available in this sector, but ad hoc education is given by nurses and doctors. The availability of dieticians depends on the individual facility. Action plans in the public sector are moving towards an integrated chronic care approach where diabetes management services will be combined with hypertension, epilepsy, asthma and other chronic diseases owing to the high rates of comorbidity in the SA population (Prof. Melvyn Freeman, personal communication).

There are distinct differences between the resources and tools available to patients treated in public as opposed to private healthcare, but it is not clear whether these differences have a significant impact on the management of diabetes. It would be very beneficial to assess whether certain treatment strategies are more effective than others in improving glycaemic control and quality of life (QoL) in children with T1DM in the SA setting.

\section{Objective}

To investigate the effectiveness of different management techniques currently being used by SA children with T1DM. Measurements of glycosylated haemoglobin ( $\mathrm{HbAlc}$ ) and QoL were used to determine the level of treatment success with regard to type of insulin administered, use of correction doses, carbohydrate counting and inclusion of regular exercise.

\section{Methods \\ Sample}

The sample consisted of 80 children diagnosed with T1DM who attended diabetes weekend camps. Five camps were randomly 
selected from different regions of SA. A diabetes nurse evaluated whether children met the inclusion criteria, which included being SA citizens, $>12$ months since the diagnosis of diabetes (in order to limit the effect of residual pancreatic function), and age 7 - 18 years at the time of data collection. All the children who were eligible for the study were contacted to ensure an equal opportunity to participate. Only children who signed assent and whose parents gave informed consent were enrolled. A cross-sectional study design was approved by the Research Ethics Committee of the University of Pretoria.

\section{Data collection}

Participants were asked to answer the Novo Nordisk Quality of Life for Youth Questionnaire, the Joslin Problem Areas in Diabetes (PAID) questionnaire, and a newly designed study questionnaire on demographics, management and perceptions of diabetes. The latter questionnaire was validated before implementation by means of a pilot study. The height and weight of each child was recorded and HbAlc measured using the A1CNow+ Multi-test system (Bayer HealthCare, USA).

\section{Data analysis}

Because the A1CNow+ system has an upper limit of detection at $13.0 \%(118 \mathrm{mmol} /$ mol), HbAlc was converted to HbAlc classification according to the American Diabetes Association guidelines. ${ }^{[3]}$ Table 1 shows that targets are slightly more stringent for adolescents. Special consideration was given to vulnerability to hypoglycaemia in younger children because of their spontaneous physical activity and their difficulty in recognising symptoms of hypoglycaemia.

Statistical analyses were performed using Stata version 12 (Stata Corporation, USA), with a two-sided significance cut-off level of $\leq 0.05$. Group comparisons for categorical variables were performed using Fisher's exact test. The Spearman rank correlation coefficient was used to evaluate the relationship between continuous variables. Student's $t$-test or the Kruskal-Wallis test was used to determine differences between continuous variables according to the data distribution profile.

\section{Results}

A summary of the demographic characteristics of the sample, diabetes treatment strategies used and any association of these variables with $\mathrm{HbA} 1 \mathrm{c}$ classification or $\mathrm{QoL}$ is shown in Table 2. Of the participants,

Table 1. HbA1c levels classified according to American Diabetes Association guidelines ${ }^{[3]}$

\begin{tabular}{lll}
\hline & \multicolumn{2}{c}{ HbAlc, \% $(\mathbf{m m o l} / \mathbf{m o l})$} \\
\cline { 2 - 3 } HbAlc classification & Children aged $7-\mathbf{1 2}$ years & Children aged 13 - 18 years \\
\hline Good & $\leq 8.0(64)$ & $\leq 7.5(59)$ \\
Fair & $8.0-10.0(64-86)$ & $7.5-10.0(59-86)$ \\
Poor & $\geq 10.0(86)$ & $\geq 10.0(86)$
\end{tabular}

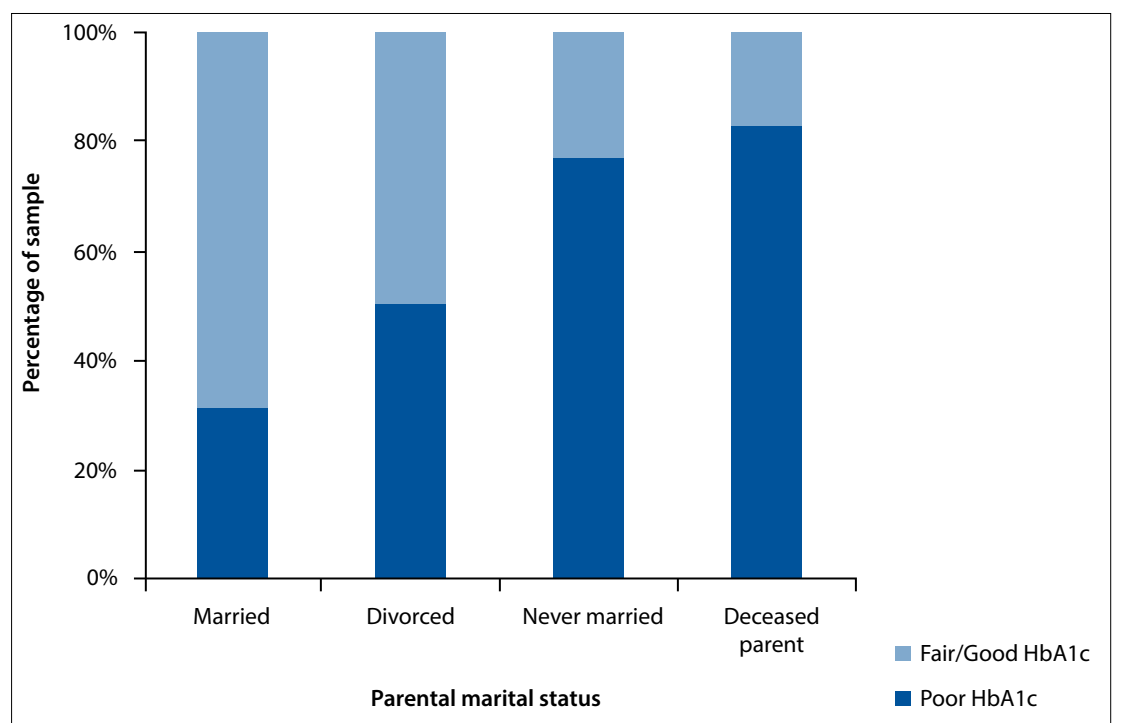

Fig. 1. Proportion of children with poor HbAlc classification compared with those with fair or good classification according to different parental marital status.

$51.4 \%$ had poor metabolic control, with an $\mathrm{HbA} 1 \mathrm{c}$ level $>10.0 \%$ ( $86 \mathrm{mmol} / \mathrm{mol})$. A significant association was found between HbAlc classification and healthcare system $(p<0.001)$, parental marital status $(p=0.002)$, insulin administration $(p=0.001)$, correction dose $(p=0.002)$, carbohydrate counting $(p<0.001)$ and number of severe hyperglycaemic events $(p=0.048)$. Fig. 1 shows the proportion of children with poor $\mathrm{HbAlc}$ in relation to parental marital status. A high score on the Novo Nordisk QoL questionnaire, on which possible scores range from 0 to 100 , indicates a high negative impact of T1DM on QoL and a low score little or no impact. The average score obtained for the sample was 28.7. The total impact of diabetes on QoL as experienced by the children was therefore not significant. However, poorer metabolic control was associated with decreased QoL, specifically in the categories of parental issues, worries about diabetes and self-rated health perception, pointing to the social consequences of poor metabolic control. According to the PAID questionnaire, an extremely low score $(0-10)$ combined with poor glycaemic control may be indicative of denial. The average score for the sample was calculated as 21.2, showing no major issues overall. A significant association was found between QoL and HbAlc classification $(p<0.001)$, healthcare system $(p<0.001)$, social designation $(p=0.009)$, parental marital status $(p=0.005)$, carbohydrate counting $(p=0.004)$ and number of severe hyperglycaemic events $(p=0.021)$.

Ten children (90.9\%) using premixed insulin were classified as having poor HbA1c control, with a significant proportion $(p<0.001)$ treated in the public healthcare system. In contrast, 24 children (54.6\%) using multiple daily injections (MDIs) had a poor HbAlc. Insulin pumps were used only in the private healthcare sector, with $3(17.7 \%)$ having poor glycaemic control. Eleven participants (84.6\%) who used no correction dose for hyperglycaemia were classified as having poor HbAlc. Thirty-five per cent were unable to give correction doses because they had been prescribed premixed insulin without separate rapid-acting insulin, while the rest used MDIs. There was a significant association between correction technique used and healthcare system $(p<0.001)$, with absence of correction more common in the public sector and correction equation more 
Table 2. Demographic characteristics of the sample, diabetes treatment regimens used and their association with HbAlc and QoL outcomes

\begin{tabular}{|c|c|c|c|}
\hline \multirow[b]{2}{*}{ Variable } & \multirow[b]{2}{*}{ T1DM children $(N=80)$} & \multicolumn{2}{|c|}{$p$-value } \\
\hline & & HbAlc & QoL \\
\hline Age (years), mean (SD) & $12.9(2.7)$ & NS & NS \\
\hline Female, \% & 56.3 & NS & NS \\
\hline BMI, median (IQR) & $18.9(15.7-21.4)$ & NS & NS \\
\hline Diabetes duration (years), median (IQR) & $4.0(2-6.3)$ & NS & NS \\
\hline Public healthcare system (\%) & 48.7 & $<0.001$ & $<0.001^{*}$ \\
\hline HbAlc classification (\%) & & - & $<0.001^{*}$ \\
\hline Good & 24.3 & & \\
\hline Fair & 24.3 & & \\
\hline Poor & 51.4 & & \\
\hline Parental marital status (\%) & & 0.002 & $0.005^{*}$ \\
\hline Married & 50.6 & & \\
\hline Divorced & 8.9 & & \\
\hline Never married & 25.3 & & \\
\hline Widowed & 15.2 & & \\
\hline Insulin administration (\%) & & 0.001 & NS \\
\hline Premixed & 13.8 & & \\
\hline Syringes & 2.5 & & \\
\hline MDIs & 62.5 & & \\
\hline Pump & 21.2 & & \\
\hline Correction dose technique (\%) & & 0.002 & NS \\
\hline No correction & 17.5 & & \\
\hline Set dose & 10.0 & & \\
\hline Sliding scale & 35.0 & & \\
\hline Correction equation & 27.5 & & \\
\hline Unknown & 10.0 & & \\
\hline Carbohydrate counting (\%) & 33.8 & $<0.001$ & $0.004^{*}$ \\
\hline Regular exercise (\%) & & NS & NS \\
\hline No physical activity & 21.5 & & \\
\hline 1 - 2 days per week for $\geq 30$ min per day & 48.1 & & \\
\hline 3 - 4 days per week for $\geq 30$ min per day & 22.8 & & \\
\hline$\geq 5$ days per week for $\geq 30$ min per day & 7.6 & & \\
\hline Severe hypoglycaemic events ( $n$ ), median (IQR) & $0(0-1)$ & NS & NS \\
\hline Severe hyperglycaemic events ( $n$ ), median (IQR) & $1(0-4)$ & 0.048 & $0.021^{\dagger}$ \\
\hline
\end{tabular}

frequently used in the private sector. A notable proportion of 36 children (89.6\%) who were not using carbohydrate counting were above the recommended targets set by the American Diabetes Association, and again a significant difference was seen between healthcare systems $(p<0.001)$.

\section{Discussion}

This study observed several factors in clinical practice that were associated with poor metabolic control and/or decreased QoL for a child with T1DM, thereby limiting the success of diabetes management. These factors included use of public healthcare, use of premixed insulin (without a correction pen), lack of correction doses, absence of carbohydrate counting, increased number of severe hyperglycaemic events and singleparent households.

Patients treated in the public healthcare system demonstrated a significant association with the use of premixed insulin $(p<0.001)$, no correction dose for hyperglycaemia $(p<0.001)$ and lack of carbohydrate counting $(p<0.001)$ compared with patients treated in private healthcare settings. Several obstacles specific to public healthcare may be responsible for these associations. The availability of testing strips in governmentfunded facilities is not standard and moreover is severely limited. Correction doses and carbohydrate counting cannot be properly used if the child cannot test his or her blood glucose at least four times a day. ${ }^{[3]}$ Currently children receive between 50 to 100 test strips per month, but this very much depends on the facility's budget and stock of test strips (Mrs Corine Verwey, personal communication). Given that an insufficient number of test strips are available, testing intensively for a few consecutive days a month may provide more useful information than twice-daily measurements and permit healthcare professionals to make informed decisions about blood glucose patterns and insulin dose adjustments. ${ }^{[4]}$ Previous research investigating the obstacles that public healthcare professionals face in managing diabetes patients listed staff shortages, budgetary constraints and the lack of culturally appropriate and simple educational material. ${ }^{[5]}$ Studies also found that an excessive patient load limited doctors' ability to provide education to patients during routine consultations, and that lack of post-basic training and a deficiency of diabetes knowledge on the part of nurses may have contributed to the poorer health outcomes of children treated at government diabetes clinics, ${ }^{[5]}$ contrasting with the availability of diabetes nurse educators in private facilities.

Almost all the children using premixed insulin were classified as having poor $\mathrm{HbAlc}$. Premixed insulin is often prescribed to children with low treatment compliance because fewer injections are needed, which may explain the very poor success seen with this insulin administration technique. However, if this is true it presents a contradiction. Children using this type of insulin require consistent carbohydrate intake to balance insulin action profile and prevent hypoglycaemia during periods of peak insulin action, especially overnight. ${ }^{[6]}$ If patients are non-compliant in taking insulin, dietary compliance cannot be expected to be high. Another reason why a patient may be prescribed premixed insulin is its relatively low cost; however, children should also have access to rapid-acting 
insulin to control hyperglycaemia and treat ketones, thus negating any cost benefit. Physicians at both private and government institutions have the ability to prescribe an MDI regimen. Previous research has shown no correlation between an increased number of daily injections and adverse QoL, ${ }^{[7]}$ and we also found no association between insulin administration and QoL. Changing to a more intensive MDI regimen would therefore facilitate improved glycaemic control without impacting negatively on QoL.

Standard formulae have been created to calculate the number of additional units of insulin requiring administration to correct hyperglycaemia, ${ }^{[8]}$ but such methods are not discussed in international guidelines for the treatment of children with T1DM. ${ }^{[3,4]}$ It is therefore not surprising that correction doses are not standardised in SA. Patients using premixed insulin without access to rapid-acting insulin have no way to correct hyperglycaemia, ensuring the failure of this insulin administration strategy and placing the child at high risk for diabetic ketoacidosis and complications of poor glycaemic control. Children who were not taking correction doses while on an MDI regimen demonstrate a lack of resource utility. They were not educated in correct hyperglycaemia management despite the availability of rapid-acting insulin that could be used to improve metabolic control. As previously mentioned, the use of correction doses is highly dependent on the availability of testing strips, and this may have influenced correction options given to the child. Should children have stable access to test strips, a tool to educate patients on correction doses is the plastic insulin dosage guide designed by Kaufman et al. ${ }^{[9]}$ This plastic card enables patients to easily determine the number of insulin units needed to correct hyperglycaemia based on current blood glucose readings. The tool can be adjusted as the child's insulin sensitivity changes, thereby including the advantages of both sliding scale and correction equation methods while accommodating children with low literacy and numeracy skills.

Carbohydrate counting was seen as the most important influence on QoL. Once a child understands how food affects their blood glucose, they are empowered to make healthy dietary decisions without feeling deprived. However, carbohydrate counting requires intensive dietary education and blood glucose monitoring, which may not be available. Lack of sufficient numeracy and literacy skills, as well as food-security issues, are further barriers to this diabetes management strategy. A more suitable method in resource-limited settings may be the use of a consistent eating plan with the flexibility to swap food items with the same carbohydrate content. ${ }^{[4]}$ This strategy could facilitate a gain in QoL while achieving improved glycaemic control without the need for additional testing strips, although comprehensive dietary education would still be necessary.

The number of severe hyperglycaemic events was seen to be significantly associated with $\mathrm{HbAlc}$ classification and QoL $(p=0.048)$. This seems intuitive, since increasing frequency of hyperglycaemia will elevate $\mathrm{HbA1c}$. It is possible that children who experienced more frequent diabetic ketoacidosis or hospitalisation with hyperglycaemia believe that their diabetes is unmanageable, thus decreasing their QoL. There was no difference in hypoglyacaemic episodes according to site $(p=0.9295)$ or insulin regimen $(p=0.5111)$.

This study did not find any association between regular exercise and $\mathrm{HbA} 1 \mathrm{c}$ classification or QoL. It was noted that little or no guidance for exercise was given to T1DM children in either public or private healthcare settings. The low proportion of T1DM children participating in sport in this study implies that exercise is not undertaken for clinical reasons but rather for social reasons or school requirements, suggesting that exercise is not likely to be maintained after school. Previous studies have failed to show improved glycaemic control with regular exercise, and the majority of the guidelines for T1DM management during exercise are based on personal experience. ${ }^{[10]}$ Despite this, exercise for patients with T1DM is considered highly beneficial, resulting in improvement in increased glucose utilisation, insulin sensitivity and weight management and a reduction in cardiovascular risk factors. ${ }^{[11]}$

It was found that children from single-parent households had decreased glycaemic control and QoL, regardless of healthcare system or management options used. The significant association found between $\mathrm{HbAlc}$ and parental marital status $(p=0.002)$ supports previous research indicating that family support is crucial in diabetes management for children. ${ }^{[12]}$ Research has shown that family members who provide high levels of support for diabetes care have children who adhere better to their diabetes regimens. ${ }^{[13]}$ Parents who anticipate imperfect blood glucose levels and use effective communication were shown to raise T1DM children without major problems. Doctors should be aware of this risk factor in their patients.

A limitation of this study was the unexpectedly low proportion of T1DM children using public healthcare (48.7\%), in contrast to $71.1 \%$ of the general SA population making use of government facilities. ${ }^{[14]}$ It is not known whether this difference is due to sampling error or representative of children living with T1DM. Selection bias may have occurred, since the sample consisted only of children who attended diabetes camps; however, these camps were free of charge to children of low socioeconomic status, and advertised to both private and public healthcare patients well in advance. Owing to lack of data on the number of children living with T1DM in SA, it was difficult to calculate the power of this study. It is currently estimated that between 5000 and 10000 children are living with T1DM in SA (Dr David Segal, personal communication). Despite these caveats, data concerning the success of various management techniques are valid and may be used to highlight clinical practices that are linked to unsatisfactory diabetes management. We believe that our results can be generalised to a large majority of SA children with T1DM, as our study included a wide range in age, duration of diabetes and ethnicity in both the public and private healthcare sectors.

We recommend that the SA National Department of Health consider implementing national policies for the treatment of T1DM in order to standardise diabetes management between healthcare systems. Such guidelines should be developed by practising healthcare professionals and academics in the field. The guidelines should be actively disseminated with the inclusion of in-service training for primary healthcare professionals in order to maximise the adoption of the new clinical standards. ${ }^{[5]}$ We also recommend that future international guidelines on the management of diabetes in children and adolescents address insulin dosing methods to correct hyperglycaemia. Greater involvement with schools is also recommended.

\section{Conclusion}

This study identified diabetes management strategies that are linked to poor glycaemic control and decreased QoL, which include use of premixed insulin without access to rapid-acting insulin, absence of correction doses for hyperglycaemia, and lack of carbohydrate counting. Treatment strategies at public healthcare facilities were 
found to be significantly less effective than methods used in the private sector. T1DM children from single-parent households were prone to unsuccessful diabetes management regardless of the treatment techniques used. Recommendations regarding the adoption of more effective diabetes management strategies within the public healthcare system are proposed.

Conflict of interest. KLK is employed by the non-profit organisation Youth With Diabetes, which gave access to the patients and donated HbAlc testing equipment; however, she played no role in initial patient recruitment. NB and SASO have no conflict of interest to declare.

Acknowledgements. The authors wish to thank Youth With Diabetes for access to patients and donation of the HbAlC testing systems. Appreciation also goes to Sr Hester Davel for her assistance in conducting the patient questionnaires.

\section{References}

1. International Diabetes Federation. Diabetes Atlas. 6th ed. Brussels: IDF, 2013. http://www.idf.org/ atlasmap/atlasmap (accessed 12 January 2014)
2. Endocrine system, diabetes mellitus. In: Pudifin D, ed. Standard Treatment Guidelines and Essential Drugs List Primary Health Care. Pretoria: Department of Health, 2008:144

3. American Diabetes Association. Standards of medical care in diabetes. Diabetes Care 2013;36(Suppl 1):S11-S66. [http://dx.doi.org/10.2337/dc13-S011]

4. Rewers $\mathrm{M}$, Pihoker $\mathrm{C}$, Donaghue $\mathrm{K}$, et al. Assessment and monitoring of glycaemic control. In: Colagiuri S, ed. Global IDF/ISPAD Guideline for Diabetes in Childhood and Adolescence. Brussels: Colagiuri S, ed. Global IDF/ISPAD Guideline for

International Diabetes Federation, $2011: 50-98$.
5. Daniels A, Biesma R, Otten J, et al. Ambivalence of primary health care professionals towards the South Daniels A, Biesma R, Otten J, et al. Ambivalence of primary health care professionals towar
African guidelines for hypertension and diabetes. S Afr Med J 2000;90(12):1206-1211.

African guidelines for hypertension and diabetes. S Afr Med J 2000;90(12):1206-1211.
Wolever TM, Hamad S, Chiasson J, et al. Day-to-day consistency in amount and source of carbohydrate intake 6. Wolever TM, Hamad S, Chiasson J, et al. Day-to-day consistency in amount and source of carbohydrate
associated with improved blood glucose control in type 1 diabetes. J Am Coll Nutr 1999;18(3):242-247.

associated with improved blood glucose control in type 1 diabetes. J Am Coll Nutr 1999;18(3):242-247. Hoey H, Aanstoot HJ, Chiarelli F, et al. Good me life in 2,101 adolescents with type

8. Davidson PC, Hebblewhite HR, Steed RD, et al. Analysis of guidelines for basal-bolus insulin dosing Basal insulin, correction factor, and carbohydrate-to-insulin ratio. Endocr Pract 2008;14(9):1095-1101. [http://dx.doi.org/10.4158/EP.14.9.1095]

9. Kaufman FR, Halvorson M, Carpenter S. Use of a plastic insulin dosage guide to correct blood glucose levels out of the target range and for carbohydrate counting in subjects with type 1 diabetes. Diabetes levels out of the target range and for carbohydrate counting in subjects w/

10. Australian Pediatric Endocrine Group. APEG Handbook on Childhood and Adolescent Diabetes. Parramatta, Australia: Child Health Promotion Unit, Royal Alexandra Hospital for Children, 1996. 11. American Diabetes Association. Physical activity/exercise and diabetes. Diabetes Care 2004;27(Suppl 1):S58-S62. [http://dx.doi.org/10.2337/diacare.27.2007.S58]
1. 5 .

2. Cameron F, Skinner T, De Beaufort C, et al. Are family factors universally related to metabolic outcomes in adolescents with type 1 diabetes? Diabet Med 2008;25(4):463-468. [http://dx.doi.org/10.1111/j.14645491.2008.02399.x.

Accepted 22 January 2015. 